\title{
Impact of surface characterizations on bone regeneration: comparison of four bone grafts in rabbit calvarial defect
}

\section{Abstract}

This study investigated the relationship between physico-chemical characterizations and biological effects of four bone grafts (HealAll, Ge-Rui, Bio-Gene, Bio-Oss) in rabbit calvarial defect. The physico-chemical characterizations of the four bone grafts were measured by special instruments separately. Four 8-mm-diameter defects were created on cranial raphe of New Zealand white rabbits and filled with Heal-All, Ge-Rui, Bio-Gene and Bio-Oss respectively. After 6 and 12 weeks, the biological effects were evaluated by microcomputed tomography (micro-CT) and histomorphometric analysis.

The biological effects of Ge-Rui are similar with those of Bio-Oss, which are both better than Heal-All and Bio-Gene. There are some correlations among nanostructures, surface area, particle size, pore size, $\mathrm{Ca} / \mathrm{P}$ ratio, organic component, calcination process for bone formation and bone graft degradation.

\section{Background and Aim}

In clinical work, there arises a great demand for bone grafts in dental implantation, bone fracture, trauma and so on. Autogenous bone is the golden standard for bone graft. Hydroxyapatite $(H A)$, $\mathrm{Ca}_{10}\left(\mathrm{PO}_{4}\right)_{6}(\mathrm{OH})_{2}$, with stoichiometric composition similar to human bone, have drawn great attention from basic researches and clinical application due to its excellent biocompatibility and bone-bonding properties. Literatures point out that the cellular esponses of the bone graft mainly depend on its physicalchemical properties, crystallinity, particle size, macroporosity, microporosity, hardness and surface structure. Based on the aforementioned background, we investigated the characterizations and the biological effects of the four bone grafts in the hope for providing theoretical basis for better choice and better manufacturing $\mathrm{HA}$ - derived bone graft in future.

The primary objective was to investigate the in vivo performance, namely in terms of the quantitative data of newly formed bone and remnant graft, of four hydroxyapatite-derived bone grafts (HA) (Heal-All, Ge-Rui, Bio-Gene, Bio-Oss) in a rabbit calvarial defect model. The secondary objective was to nvestigate the correlations between the surface characterizations and in vivo biological performance.

\section{Methods and Materials}

Four HA-derived bone grafts (Heal-All, Ge-Rui, Bio-Gene, Bio-Oss) were used for the surgeries as delivered by four different manufacturers. The surface characterizations were evaluated by SEM, XPS, XRD, FT-IR and BET pore analyzer. Four 8-mm-diameter defects were created on the left, right, up and down of the crania raphe of New Zealand White rabbit and filled with Heal-All, Ge-Rui, Bio-Gene and Bio-Oss respectively. The samples were harvested at 6 and 12 weeks after surgery and inspected by Micro-CT and histological and histomorphometric evaluations.

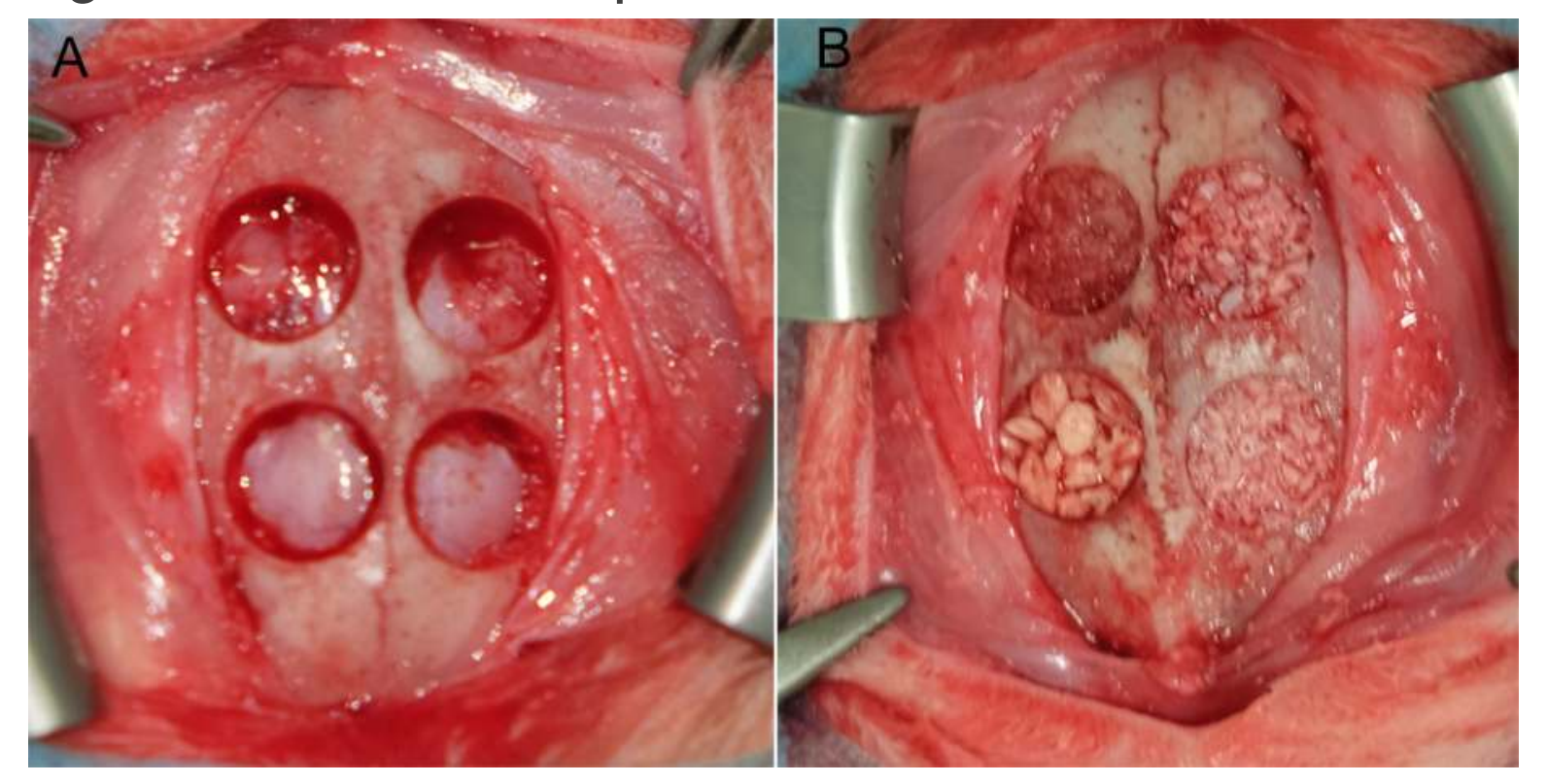

\section{Results}

The topographies of Bio-Oss and Ge-Rui with more granules were rougher than those of Heal-All and Bio-Gene. The main component of the four bone grafts was HA. And the four bone grafts all mainly contained $\mathrm{O}, \mathrm{Ca}$ and $\mathrm{P}$ element, while Heal-All and BioGene also contained organic N element. The specific surface area, BJH Adsorption average pore volume and size of Ge-Rui and BioOss were significantly larger than those of Heal-All and Bio-Gene. Micro-CT analysis demonstrated that the percentage of newly formed bone in Ge-Rui (37.308 \pm 3.401$)$ and Bio-Oss (40.026 \pm 4.066$)$ defects were significantly more than that in Bio-Gene

$(23.726 \pm 5.713)(P<0.05)$ and Heal-All $(29.175 \pm 6.656)(P<0.05)$ defects at 6 weeks. And at 12 weeks, the percentage of newly formed bone in Bio-Oss $(40.089 \pm 5.066)$ defect was significantly more than that in Bio-Gene (25.844 44.461$)$ and Heal-All $(30.42 \pm 7.298)$ defects $(P<0.05)$. Histological observation showed that most of the new bone and Tartrate-resistant acid phosphatase (TRAP) positive cells were scattered in the fibrous connective tissue, rarely wrapping the graft in Heal-All defect. However, in the rest of three graft defects, the newly formed bone and TRAP positive cells were concentrated around the grafts, wrapping the grafts. Histomorphometric evaluations showed that the quantity of TRAP positive cells in Heal-All defect was significantly more than that in Bio-Gene and Bio-Oss defects at 6 and 12 weeks $(P<0.05)$

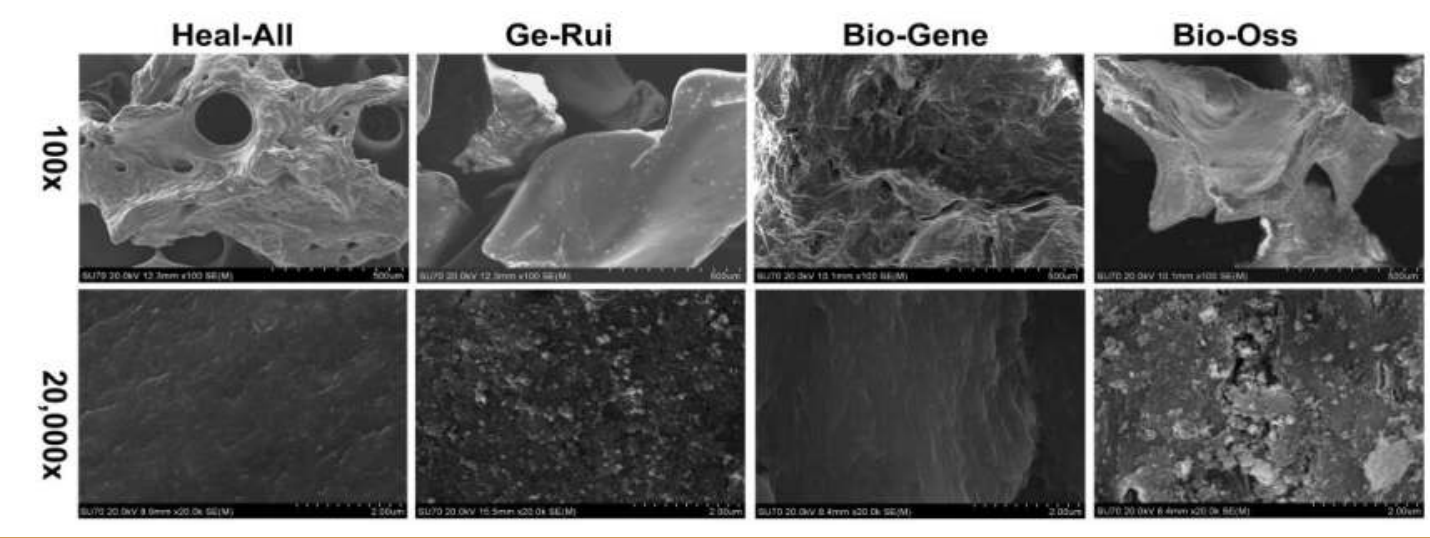

Conclusions

Four kinds of HA-derived bone grafts displayed different characterizations and in vivo bone regeneration properties. The biological effects of Bio-Oss are the best. The biological effects of Ge-Rui are similar with those of Bio-Oss, which are both better than Heal-All and Bio-Gene in the rabbit calvarial defect model. Nanostructures, bigger surface area, suitable particle size and suitable pore size of the bone grafts can effectively promote new bone formation and further enhance bone regeneration. There are some correlations among bone graft degradation and particle size, $\mathrm{Ca} / \mathrm{P}$ ratio, organic component, calcination process and so on.
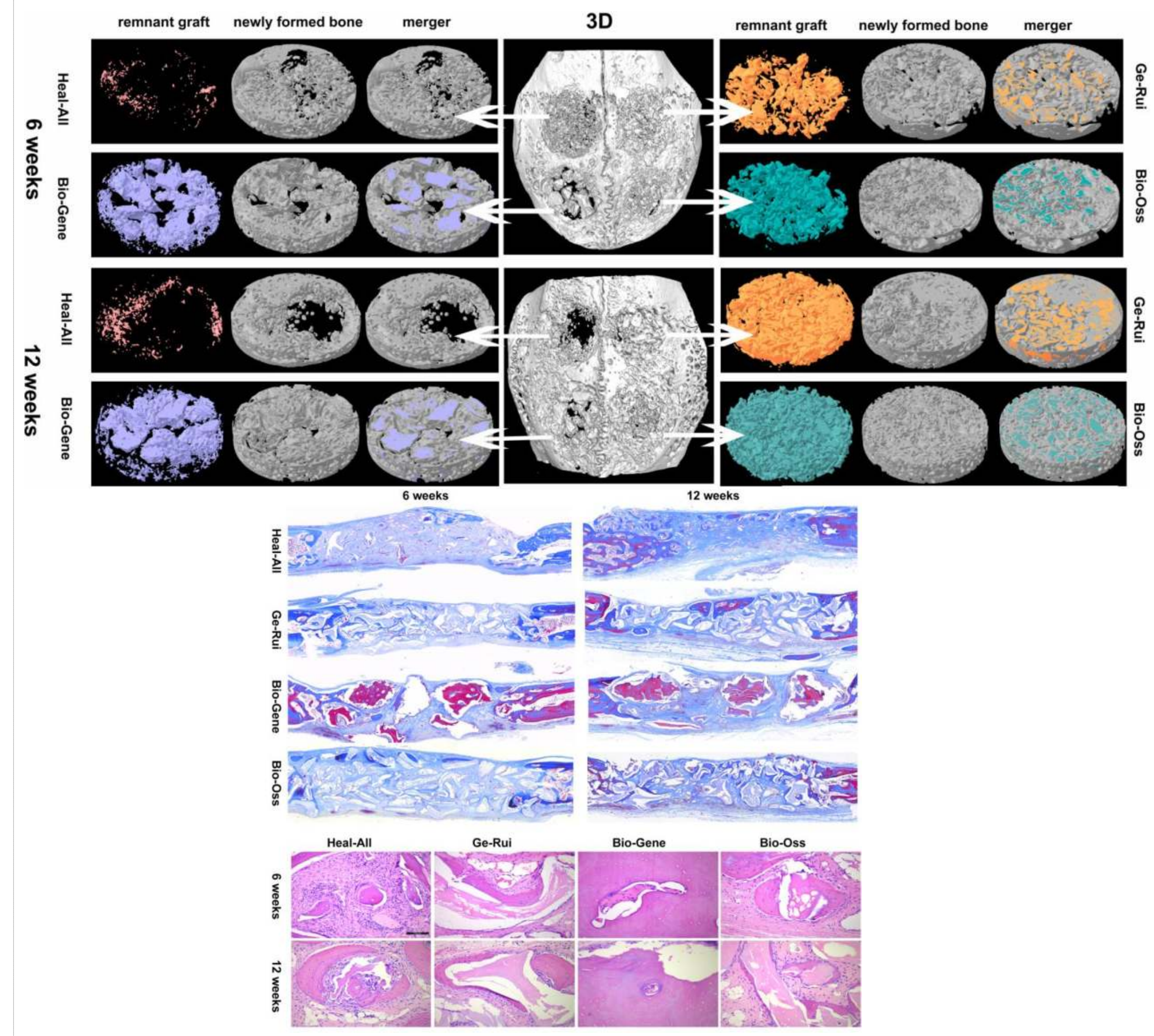

References

1.Autogenous regulation of a network of bone morphogenetic proteins (BMPs) mediates the osteogenic differentiation in murine marrow stromal cells. Bone.

2.Osteogenic potential of autogenous bone grafts harvested with four different surgical techniques Journal of Dental

3.Impact of Bone Harvesting Techniques on Cell Viability and Cinical implant dentistry and related re 